\title{
PAPER
}

\section{The interaction between acoustic salience and language experience in developmental speech perception: evidence from nasal place discrimination}

\section{Chandan R. Narayan, ${ }^{1}$ Janet F. Werker ${ }^{2}$ and Patrice Speeter Beddor ${ }^{3}$}

1. Department of Humanities, University of Toronto Scarborough and Graduate Faculty of Linguistics, University of Toronto, Canada

2. Infant Studies Centre, Department of Psychology, University of British Columbia, Canada

3. Department of Linguistics, University of Michigan, USA

\begin{abstract}
Previous research suggests that infant speech perception reorganizes in the first year: young infants discriminate both native and non-native phonetic contrasts, but by 10-12 months difficult non-native contrasts are less discriminable whereas performance improves on native contrasts. In the current study, four experiments tested the hypothesis that, in addition to the influence of native language experience, acoustic salience also affects the perceptual reorganization that takes place in infancy. Using a visual habituation paradigm, two nasal place distinctions that differ in relative acoustic salience, acoustically robust labial-alveolar [ma]-[na] and acoustically less salient alveolar-velar [na]-[na], were presented to infants in a crosslanguage design. English-learning infants at 6-8 and 10-12 months showed discrimination of the native and acoustically robust [ma]-[na] (Experiment 1), but not the non-native (in initial position) and acoustically less salient [na]-[na] (Experiment 2). Very young (4-5-month-old) English-learning infants tested on the same native and non-native contrasts also showed discrimination of only the [ma]-[na] distinction (Experiment 3). Filipino-learning infants, whose ambient language includes the syllable-initial alveolar $(/ \mathrm{n} /)$-velar $(/ \mathrm{y} /)$ contrast, showed discrimination of native [na]-[na] at 10-12 months, but not at 6-8 months (Experiment 4). These results support the hypothesis that acoustic salience affects speech perception in infancy, with native language experience facilitating discrimination of an acoustically similar phonetic distinction [na]-[na]. We discuss the implications of this developmental profile for a comprehensive theory of speech perception in infancy.
\end{abstract}

\section{Introduction}

The past 35 years of research into developmental speech perception has detailed the remarkable abilities infants bring to the language-learning table before they utter their first word (for reviews see Jusczyk, 1997; Kuhl, 2004; Saffran, Werker \& Werner, 2006). Of particular interest for the present work is the early finding that young infants are able to discriminate speech sounds that do not occur in their native language (e.g. Eimas, Siqueland, Jusczyk \& Vigorito, 1971; Streeter, 1976; Eilers, Wilson \& Moore, 1977). In comparison, adults have difficulty discriminating most non-native contrasts, including contrasts that infants from the same language community discriminate (e.g. Trehub, 1976; Werker, Gilbert, Humphrey \& Tees, 1981). Adult discrimination of non-native speech contrasts is constrained by the set of distinctive speech sounds of their native language (for reviews see Strange, 1995, and Sebastián-Gallés, 2005), a filtering system that very young infants have not yet developed. Over the course of their first year, however, infants' speech perception undergoes a well-known reorganization, such that sensitivity to those previously discriminable non-native contrasts typically declines in the absence of language experience. For example, 6-8month-old English-learning infants discriminate nonnative place of articulation contrasts, such as the dentalretroflex stop contrast in Hindi or the velar-uvular ejective contrast in the Pacific Northwest language Nthlakampx, but 10-12-month-old English learners do not (Werker \& Tees, 1984; Anderson, Morgan \& White, 2003). That 10-12-month-old infants from Hindiand Nthlakampx-speaking households successfully discriminate their respective native contrasts suggests that native-language input serves to maintain the sensitivity that was initially present. A similar pattern of perceptual development has been found with other oral consonant contrasts (e.g. Werker et al., 1981; 
Tsushima, Takizawa, Sasaki, Shiraki, Nishi, Kohno, Menyuk \& Best, 1994; Best, McRoberts, LaFleur \& Silver-Isenstadt, 1995; Rivera-Gaxiola, Silva-Pereyra \& Kuhl, 2005), lexical tone (Mattock \& Burnham, 2006), sign language (Baker, Michnick-Golinkoff \& Petitto, 2006), and vowel contrasts (e.g. Cheour, Alho, Ceponiene, Reinikainen, Sainio, Pohjavuori, Aaltonen $\&$ Naatanen, 1998). The last of these shows an earlier age of reorganization (6-8 months as opposed to 10 12 months for consonants; Kuhl, Williams, Lacerda, Stevens \& Lindblom, 1992; Polka \& Werker, 1994).

More recently, the reorganization of infants' perceptual sensitivities in the first year of life has been shown to be more nuanced than the findings of experience-based discrimination maintenance or decline would suggest. An accumulating body of literature suggests that the discrimination of certain consonantal contrasts improves with language-specific experience. For example, by the end of their first year, experience with Mandarin improves Mandarin-learning infants' discrimination of the native affricate-fricative $\left(/ \mathrm{tc} 6^{(\mathrm{h})} /{ }^{1}\right.$ /6/) contrast (Tsao, Liu \& Kuhl, 2006). Similarly, by the age of 12 months, experience with English improves English-learning infants' discrimination of the native / $\mathrm{r}$ / $-1 /$ contrast (Kuhl, Stevens, Hayashi, Deguchi, Kiritani \& Iverson, 2006; see Sebastián-Gallés, 2006, for discussion). Language experience facilitates discrimination of the $/ \mathrm{d} /-/ \mathrm{d} /$ contrast into early childhood and adulthood for English-learning children (Polka, Colantonio \& Sundara, 2001; Sundara, Polka \& Genesee, 2006). In all of these studies demonstrating facilitation (Polka et al., 2001; Kuhl et al., 2006; and Tsao et al., 2006), the discrimination performance of young infants on the native contrast was good (significantly above chance), but not as good as the performance of older infants whose results approached the performance of adults on the same contrasts. In addition to enhancing or facilitating the discrimination of native contrasts, language experience has also been shown to realign initial sensitivities by the end of an infant's first year so that they better match native phonetic category distinctions (Burns, Yoshida, Hill \& Werker, 2007). Thus, the emerging picture of the reorganization of infant speech perception encompasses a variety of experiencerelated profiles that begin with successful discrimination of language-general phonetic contrasts in early infancy and develop into a more refined perceptual sensitivity reflecting the speech contrasts of the infant's ambient language by the end of their first year and beyond.

Not all phonetic contrasts fit this developmental profile, however. For example, some non-native phonetic contrasts remain highly discriminable across

\footnotetext{
'Throughout the paper, phonetic symbols are given in brackets ('[ ]') and phonemes given between slashes ( $/ /$ ') according to convention in the linguistics literature.
}

development. Best, McRoberts and Sithole (1988) showed that 12-14-month-old English-learning infants and English-speaking adults discriminate non-native place of articulation contrasts in clicks (sounds produced by releasing a pocket of air trapped by the tongue in the mouth found in some languages of southern Africa). They argued that the characteristics of click contrasts were sufficiently different from English contrasts that they were discriminable by older infants even in the absence of native-language experience. Certain non-native vowel contrasts also remain discriminable across development. In their study of cross-language vowel perception, Polka and Bohn (1996) found that the German front-back high vowel contrast $/ \mathrm{y} /-/ \mathrm{u} /$ remained discriminable across English-learning infants' development, failing to replicate Polka and Werker's (1994) demonstration of language-specific reorganization for these vowels by 6-8 months. Polka and Bohn (1996) suggested that the contrast remained discriminable for English-learning infants for acoustic reasons: the formant differences distinguishing the $/ \mathrm{y} / \mathrm{-} / \mathrm{u} /$ contrast in their study were substantially larger than the formant differences observed in the stimuli used by Polka and Werker (1994).

In comparison, whereas some contrasts are discriminable without native experience, recent evidence indicates that having relevant language experience may not be sufficient for some phonetic contrasts to remain discriminable across development. For example, Bosch and Sebastián-Gallés (2003) showed that infants growing up bilingual in Spanish and Catalan discriminate the Catalan front tense-lax vowel contrast $/ \mathrm{e} /-/ \varepsilon /$ at 4 and 12 months but not at 8 months. The authors suggest that one factor contributing to these infants' exceptional developmental pattern for the $/ \mathrm{e} /-/ \varepsilon /$ contrast is the acoustic similarity, in perceptually relevant first and second formant $(\mathrm{F} 1 \times \mathrm{F} 2)$ space, between the two Catalan vowels and the single Spanish vowel in this region of the space. That is, the acoustic similarity of the vowels in bilingual exposure, coupled with the emerging heightened sensitivity to contrastive vowels at 8 months, may result in weakened $/ \mathrm{e} /-/ \varepsilon /$ discrimination at this age. Recently, the authors have extended this general finding to include the acoustically similar $/ \mathrm{o} / \mathrm{-} / \mathrm{u} /$ contrast. Spanish-Catalan bilinguals fail to discriminate the Spanish/Catalan $/ \mathrm{o} /-/ \mathrm{u} /$ contrast at 8 months, but successfully discriminate the acoustically more distinct Spanish/Catalan /e/-/u/ contrast (Sebastián-Gallés and Bosch, in press).

Other findings also suggest that acoustic salience interacts with experiential factors in infant speech perception. In early work, Eilers et al. (1977) showed that native contrasts involving $/ \mathrm{f} /, / \theta /$ ('th'), and $/ \mathrm{J} /$ ('sh') prove difficult for young English-learning infants, possibly due to acoustic similarity. Aslin, Pisoni, Hennessy and Perey (1981) found that although English-learning infants reliably discriminated voice 
onset time $(\mathrm{VOT})^{2}$ differences between stop consonants in both negative (non-native) and positive (native) VOT regions, their performance was significantly better when VOTs were positive; coupled with similar results from adult discrimination of pure tone signals analogous to VOT (Pisoni, 1977), they attributed infants' lower sensitivity to distinctions in the negative VOT region to acoustics. Much more recently, Polka et al. (2001), in discussing the facilitation of native contrasts with increasing linguistic experience, speculated that young infants are poorer than older infants at discriminating [d] and [ð] due to the acoustic weakness of these sounds (i.e. these sounds have an overall low amplitude rendering them difficult to discriminate). Further, the accurate discrimination of clicks by older English-hearing infants (Best et al., 1988) may be related to especially robust acoustic differences, although acoustic measures are needed to support this speculation.

That acoustic salience affects infants' discriminative abilities is in keeping with the theoretical treatment of the development of speech perception offered by Aslin and Pisoni (1980) (cf. Burnham, 1986). Under the rubric of attunement theory, Aslin and Pisoni (1980) viewed the discrimination of phonetic contrasts as being influenced by both experiential and psychoacoustic factors, which allow for different routes to mature perception for different contrasts (e.g. maintenance and loss as shown by Werker \& Tees, 1984, and Best et al., 1995; facilitation as shown by Polka et al., 2001, and Kuhl et al., 2006). Moreover, adult perception is also affected by acoustic salience. For example, Polka (1991) and Pruitt (1995) have shown that the discriminability of the Hindi dentalretroflex contrast for English speakers varies significantly as a function of voicing, which can enhance the perceptibility of the non-native contrast.

Given the wide variety of phonetic gestures employed by the world's languages for contrasting speech sounds, and their diverse acoustic consequences, it is expected that certain phonetic contrasts are inherently perceptually easier to differentiate than others and in turn affect experientially shaped patterns of perceptual reorganization across development. The present study explores the precise nature of this interaction by examining the development of infants' discrimination of two pairs of phonetic contrasts that differ in their relative acoustic salience. We ask whether a less salient contrast whose realizations are close in perceptually relevant acoustic space shows a developmental pattern different from that of a more salient contrast whose realizations are further apart in the same acoustic space.

\footnotetext{
${ }^{2}$ VOT is the duration between the release of an oral occlusion for the production of a consonant and the onset of periodic vibration of the vocal cords for the production of the post-consonantal vowel. American English speakers exhibit voicing contrasts (such as the difference between ' $p$ ' and ' $b$ ') in the positive (post-release) region of the VOT dimension.
}

We approach this question by considering infants' perception of native and non-native nasal place of articulation contrasts. Nasal consonants are speech sounds such as $[\mathrm{m}]$ and $[\mathrm{n}]$ in which the velum is lowered, giving rise to resonances in the coupled nasal and oral cavities, as compared to oral consonants, such as $[\mathrm{p}]$ and $[\mathrm{t}]$, whose acoustic resonances are oral. Although considered consonants phonetically and phonologically, nasal consonants exhibit different phonological behavior from orals as evidenced by their asymmetric distribution, in comparison to orals, in the sound systems of the world's languages. While an overwhelming majority $(\sim 99 \%)$ of the world's languages have oral consonants produced with bilabial $(/ \mathrm{p} /)$, alveo-dental $(/ \mathrm{t} /)$, and velar $(/ \mathrm{k} /)$ constrictions, only about half of the world's languages have corresponding nasal consonants at all three places of articulation, with languages having bilabial $/ \mathrm{m} /$ and alveo-dental $/ \mathrm{n} /$ more often than velar $/ \mathrm{y} /$ (i.e. ' $\mathrm{ng}$ ' as in 'sing') (Maddieson, 1984). The distribution of these sounds within syllables shows an even greater asymmetry. Languages are five times more likely to lack $/ \mathrm{y} /$ in syllable-initial (onset) position than in syllable-final (coda) position (Anderson, 2005), while $/ \mathrm{m} /$ and $/ \mathrm{n} /$ are generally not subject to such restrictions. For example, whereas Filipino has all three nasals in both positions, English has all three nasals in coda position (like 'Pam', 'pan', and 'pang') but only $/ \mathrm{m} /$ and $/ \mathrm{n} /$ in onsets ('map', and 'nap', but not 'ngap'). Thus the contrast between $/ \mathrm{n} /$ and $/ \mathrm{y} /$ is native for English speakers in final, but not in initial, position.

Linguists have proposed that acoustic salience is a factor influencing the distribution of sounds in phonological systems (Liljencrants \& Lindblom, 1972; Lindblom, 1986), with some researchers speculating that the relatively high frequency of certain contrasts, like onset $/ \mathrm{m} /-/ \mathrm{n} /$, is linked to their being acoustically more salient than less common contrasts, like onset $/ \mathrm{n} /-/ \mathrm{p} /$ (Maddieson, 1984, p. 15). If the phonological distribution of nasals reflects their perceptual salience, we would expect differences between syllable-onset $/ \mathrm{m} /$ and $/ \mathrm{n} /$ to be more salient than those between $/ \mathrm{n} /$ and $/ \mathrm{y} /$, and the results of a recent acoustic and perceptual study with Filipino nasals are consistent with this hypothesis. Narayan (2008) analyzed the spectral characteristics of the monosyllables [ma], [na], and [na] produced by three Filipino speakers. Measures of the distance between nasals' second and third formant frequencies (F2 and F3) at the juncture between the nasal murmur and the following vowel, as well as dynamic energy measurements, showed that [na] productions were spectrally closer to [na] than to [ma] tokens, especially in F2 space. A discriminant function analysis of the acoustic data showed $20 \%$ confusion between the [na] and [na] tokens, whereas tokens from the [ma] category were predicted nearly perfectly $(97 \%$ correct).

Additionally, perceptual experiments by Narayan (2008) with adult listeners showed that the nasal 
comparison with the larger acoustic distances, [m]-[n], was perceptually more salient. English-speaking adults showed near ceiling discrimination of the native [ma][na] distinction, but chance performance on [na]-[ya] (see also Larkey, Wald \& Strange, 1978, for synthetic [næ][yæ]). Although the magnitude of difference was considerably smaller, Filipino-speaking adults also showed slightly but significantly better discrimination of [ma]-[na] than [na]-[na] and, when tested on these contrasts in noise, they maintained [ma]-[na] discrimination while [na]-[na] dropped to near chance. That differences between some nasal place contrasts are more detectable than others, for both native and nonnative adult listeners, has also been shown by Harnsberger (2001). ${ }^{3}$

Our predictions for tests of infants' perception of native and non-native nasal place contrasts that differ in relative acoustic salience were guided throughout by the general reorganization patterns of development seen in oral consonant perception, with an eye towards the potential effects of acoustic salience on infant perception as seen in previous infant studies (e.g. Best et al., 1988; Polka et al., 2001; Bosch \& Sebastián-Gálles, 2003). The acoustic salience hypothesis predicts that infants' discrimination of the less salient (Filipino) [na]-[na] would be less accurate than their discrimination of the more salient (Filipino) [ma]-[na]. The age ranges and methods employed in these experiments followed closely from previous research in cross-language developmental speech perception, the goal of which was to identify both initial biases and experiential influences in infants' discrimination of native and non-native phonetic contrasts.

Using a visual habituation task, Experiments 1 and 2 test English-learning infants at 6-8 and 10-12 months on discrimination of the native [ma]-[na] and non-native [na]-[na], while Experiment 3 extends testing to 4-5month-old English-learning infants. Previewing the findings, English-learning infants of all age groups show discrimination of the native [ma]-[na] distinction but not the non-native [na]-[na], differing from the more typical pattern in the literature of phonetic contrast discrimination by younger infants. Experiment 4 tests Filipino-learning infants on native [na]-[na], which reveals a developmental profile whereby young infants do not reliably show discrimination of the contrast that older infants discriminate. We argue that nasal place contrasts are broadly represented according to robust acoustic salience in young infants, with facilitation of finer-grained phonetic distinctions emerging only later in the infant's first year.

Nasal consonants have not received the attention that oral consonants (Werker et al., 1981; Werker \& Tees, 1984; Tsushima et al., 1994; Best et al., 1995; Nittrouer \&

\footnotetext{
${ }^{3}$ Harnsberger (2001) investigated non-native nasal perception in speakers of Indo-Aryan and Dravidian languages, neither of which exhibits the velar nasal in syllable onsets.
}

Miller, 1997; Polka et al., 2001; McMurray \& Aslin, 2005; Rivera-Gaxiola et al., 2005; Kuhl et al., 2006; Burns et al., 2007) and vowels (Kuhl et al., 1992; Polka \& Werker, 1994; Polka \& Bohn, 1996; Cheour et al., 1998) have received in the cross-language developmental speech perception literature. Hillenbrand's (1984) investigation of 5.5-6.5-month-old infants' perception of bilabial and alveolar nasals across various vowels and speaker contexts is thus far the only study to assess nasal place of articulation discrimination in infancy. Using the conditioned head turn task, Hillenbrand reported that infants, trained to discriminate [ma]-[na] as spoken by a male speaker, were able to transfer their knowledge of nasal place to novel speaker genders and vowel contexts. Hillenbrand's work clearly shows that young Englishhearing infants can discriminate native [ma]-[na], a finding that is replicated in Experiment 1 using a more passive task that does not reward the infant for discrimination as in conditioned head turn.

\section{Experiment 1}

Experiment 1 tested English-learning infants at 6-8 and 10-12 months on the native [ma]-[na] distinction as produced by a speaker of Filipino. (Although the tokens were non-native, Filipino [m] and [n] are like their English counterparts and sound English-like to adult speakers of English.) Infants were expected to discriminate the contrast; failure to discriminate would be surprising and likely point towards a general difficulty for infants to discriminate nasal place (or to inappropriateness of the discrimination task). This prediction was guided by Hillenbrand's (1984) results, and also by an acoustic salience hypothesis that predicts that phonetic tokens that are distant in perceptually relevant space will be highly discriminable across development. In addition, an experiential account would predict that native place of articulation contrasts (here the [ma]-[na] contrast for English-learning infants) are discriminated across the first year of development and into adulthood.

\section{Methods}

\section{Assessment of discrimination: visual habituation}

Infants' discrimination performance was assessed using the 'switch' variate of the visual habituation $(\mathrm{VH})$ paradigm. We used this procedure for assessing speech discrimination because of its applicability to a wide range of ages of infants as well as the ease and speed of its execution (Werker, Shi, Desjardins, Pegg, Polka \& Patterson, 1998; see Kajikawa, Fais, Mugitani, Werker \& Amano, 2006, for an identical implementation with infants of 6,12 , and 18 months). The procedure begins with the presentation of auditory stimuli, which is contingent upon the infant's visual fixations on a visual pattern. Habituation to the auditory 'background' 
stimuli is indexed by a decrement in looking time (LT) to some predetermined criterion, at which point a shift to a new set of auditory stimuli occurs. Discrimination between the background and shift stimuli is determined by a significant postshift recovery in LT to the same visual target. (For other implementations of $\mathrm{VH}$ to test speech discrimination see Best et al., 1988; Pegg, Werker \& McLeod, 1992; Polka \& Werker, 1994; Best et al., 1995; and Burns et al., 2007.) In our current implementation of the procedure, LT was calculated over groups of three 14-second trials and the criterion for habituation was set at a decrement in LT of $40 \%$ for the longest three consecutive trials. Auditory presentation was not contingent on infants' looking; rather, auditory stimuli were presented for a full 14 seconds per trial whether the infant was looking or not. Infants received two post-habituation test trials, one 'same' trial in which the individual tokens were the same as the tokens in the habituation trial but in a different order, and one 'change' trial in which tokens that were different phonetic categories from the habituation tokens were presented. The 'same' and 'change' trials were counterbalanced in presentation.

\section{Participants}

Infants were recruited from a database at the University of British Columbia. Data from 32 infants were analyzed in Experiment 1, 16 infants (nine females, seven males) aged $10-12$ months $($ mean $=303$ days, range $=291$ to 332 days) and 16 (eight females, eight males) aged 6-8 months (mean $=211$ days, range $=177$ to 241 days). An additional 10 babies participated in the study, but were excluded from analysis due to crying or fussiness $(n=5)$, parental interference $(n=2)$, or failing to reach the strict habituation criterion $(n=3)$ described in detail below (Procedure). Infants were included in the analysis only if they habituated within nine to 24 trials and had LTs longer than 1 second during each test trial. Of the infants excluded due to the habituation criteria, two infants (aged 10-12 months) habituated too quickly and one infant (6-8 months) failed to habituate within 24 trials. All the infants who participated were being exposed to at least $90 \%$ Canadian English according to parental reports.

\section{Stimuli}

Three tokens of each nasal place ([ma], [na] and [ya]) were selected from a larger set of recordings made by a female native speaker of Filipino. The monosyllables were read with a level intonational contour. Figure 1 plots the F2 and F3 frequencies of the speaker's larger set of tokens of [ma], [na], and [na] (as measured at the nasal-vowel juncture). A discriminant analysis of the tokens showed $87.5 \%$ correct classification of the entire set of tokens, with $100 \%$ correct classification of [ma] tokens; [na] tokens were incorrectly classified as [na] $25 \%$ of the time, while [na]

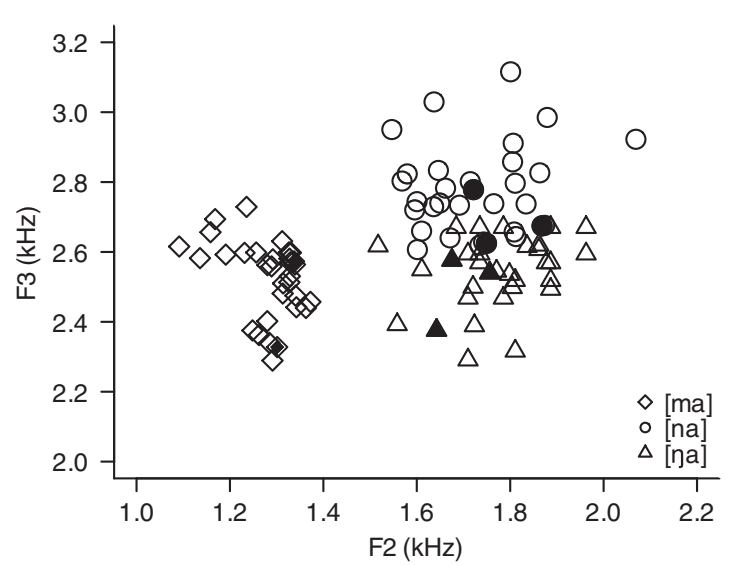

Figure 1 F2 and F3 frequencies (measured at the NV juncture) for multiple (30-32) repetitions of [ma], [na], and [ya] produced by the Filipino speaker. Filled symbols represent stimuli used in the experiments.

tokens were incorrectly classified as [na] $9.4 \%$ of the time. This speaker's pattern of overlap between [na] and [na] tokens in the perceptually relevant $\mathrm{F} 2 \times \mathrm{F} 3$ space was similar to a discriminant analysis of [ma], [na], [na] spoken by a larger set of speakers (Narayan, 2008). The speaker's tokens were also analyzed in a perceptual space representing the dynamic energy change between the nasal murmur and the following vowel (rms energy change in Bark 5-7 × rms change in Bark 11-14). Similar results were obtained, with clear separation between tokens of [ma] and those of both [na] and [na], but with overlap between [na] and [na] tokens.

The three stimuli for each place were selected based largely on minimizing acoustic differences - both within and across categories - viewed as non-systematic variation not linked to place of articulation differences. Thus tokens were selected that differed minimally in the fundamental frequency $\left(f_{0}\right)$ of the nasal murmur and vowel as well as in the overall pitch contour. Additionally, to the extent possible, non-systematic variation in the vocalic portion at vowel midpoint (and beyond) of the stimuli was avoided in order to preclude discrimination based on vowel quality. The nine stimuli (three per place) that were best matched for these characteristics were then presented for identification to an adult native Filipino speaker (who was not the speaker who produced the tokens), and each was reliably identified as the intended stimulus. Table 1 shows the acoustic characteristics of the nine stimuli used in Experiment 1 and subsequent experiments; the filled points in Figure 1 graphically represent these stimuli in F2 $\times$ F3 space. Nasal-place measurements (F2 and F3) were taken from a $10 \mathrm{~ms}$ analysis window centered at the zero-crossing between the final pulse of low-frequency periodicity of the nasal murmur and the first pulse of vowel periodicity. Vowel quality measurements (F1 and F2) were taken at vowel midpoint.

Only alveolar ([na]) and bilabial ([ma]) tokens were presented in Experiment 1. Trials (either habituation or 
Table 1 Nasal stimuli acoustics. F2 and F3 onset measurements were made at the nasal-vowel juncture, while F1 and F2 midpoint measurements were made at the midpoint of the [a] vowel. Boxed averages represent critical spectral dimensions distinguishing nasal place of articulation

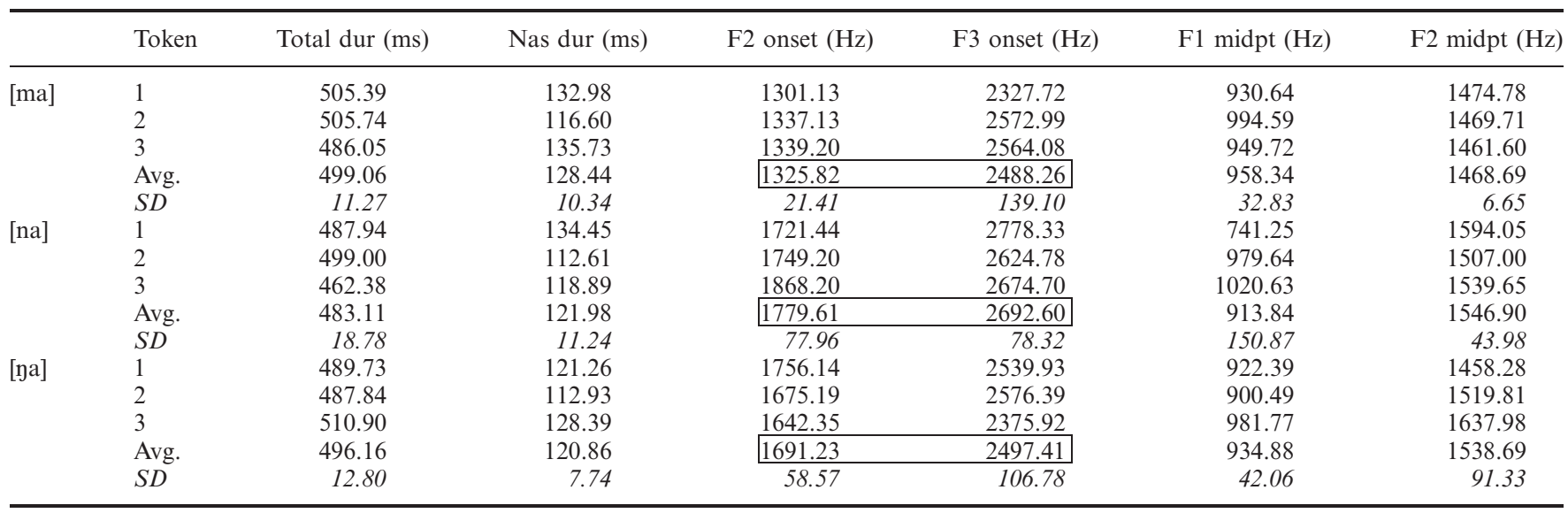

test) were approximately 14 seconds long. Two trial sets were assembled for both [na] and [ma] stimuli. Each trial consisted of the three acoustically different tokens of the stimulus category repeated three times, for a total of nine randomly ordered tokens. The two trials for each stimulus category consisted of the same tokens, but in a different random order. The inter-stimulus interval within each trial was approximately $1000 \mathrm{~ms}$.

\section{Setup and apparatus}

All infant studies were conducted in a quiet room measuring approximately $9^{\prime} \times 8^{\prime}$. Infants sat on their caregiver's lap, who was seated in a chair approximately $4^{\prime}$ from a $27^{\prime \prime}$ color TV monitor. The monitor was approximately $3.5^{\prime}$ off the ground. Below the monitor were an amplifier and two Bose 101 speakers which were approximately $30^{\circ}$ to the left and right of centerline of the caregiver's chair. A small JVC video camera (GXN7UT) stood on a tripod directly below (approximately $\left.1^{\prime}\right)$ the TV monitor.

The amplifier was connected to an Apple Macintosh G4 (OSX) computer in the adjacent control room. The Habit 2002 software program (Cohen, Atkinson \& Chaput, 2004) was used to present both visual and auditory stimuli, as well as record the infant's on-line LT. The video camera provided a closed-circuit broadcast of the infant's face to a monitor in the control room. The observer would watch the infant on the control room monitor and record 'looks' to the TV monitor in the testing room on the Macintosh computer.

\section{Procedure}

Audio stimuli were presented free-field at $70 \pm 2 \mathrm{~dB}$ SPL. On-line LT was recorded by depressing a designated key on the computer keyboard whenever the experimenter determined that the infant was looking at the TV monitor image. These looks were recorded by the Habit program and used to calculate habituation.
Habituation was determined by calculating the infant's LT to the unbounded image of a red and black checkerboard over a window of three trials. The program calculated the LT for successive three-trial windows, and habituation was achieved when a window fell to $60 \%$ of the looking time (a decrement of $40 \%$ ) of the longest three-trial window. With this nonoverlapping window, habituation occurred at $6,9,12$, $15,18,21$, or 24 trials.

Given the setup of the Habit program, a random depression in looking time during a given trial (due to dropping a toy or sneezing, for example) in the habituation period could potentially trigger the program to start the test trials. For example, if the infant sneezed during a habituation trial and as such closed her eyes for a long period prior to the sneeze, the LT could deceptively fall to $60 \%$ of the looking time of the longest window of three trials, signaling habituation. For this reason, an a priori decision was made to exclude from the analysis any infants who habituated to the background stimulus in six trials. This ensured that infants minimally heard the background stimulus for approximately 2 minutes. If an infant's LT to any given trial (habituation or test) was less than 1 second, the trial was repeated. Infants were excluded from analysis if their LT to any test trial was under 1 second. Additionally, some infants were excluded from analysis due to their failure to habituate within 24 trials, the maximum number of habituation trials given in the Habit program.

The experiment began with a pretest and ended with a posttest assessment of the infant's interest in the task in general. The visual stimulus for these tests was an animated spinning waterwheel. This image was accompanied by short tokens of a randomly rising and falling sine wave tone. If infants' LTs to the posttest were significantly shorter than their LT to the pretest, then the reliability of the habituation would be in question for it indicates a lack of interest or participation in the task in general. Trials were separated with a visual 'attention getter' consisting of an animated circular sunburst 
against contrasting backgrounds. The attention getter was not accompanied by an auditory stimulus. When the infant re-fixated on the attention getter, the experimenter would begin the next trial. Half the infants were habituated to [ma], with [na] as the change trial and [ma] as same, and half were habituated to [na].

Off-line coding. For each infant who completed the study (i.e. completed the posttest without crying, habituated within nine to 24 trials, and did not repeat test trials) their accompanying video tape was digitized to QuickTime (Apple Inc.) format using the video editing software FinalCut Pro for the Macintosh computer. These digitized files were then coded by the experimenter who was unaware of which test trial was a change or same trial. The digitization process rendered the video file at a resolution of 30 frames per second. The experimenter proceeded through the video file, frame-by-frame, logging infant looks and non-looks. This off-line coding was thought to be more reliable than the on-line coding conducted during the experiment, whose only use was to determine habituation. For every participant off-line results were compared to on-line data. In every case offline results reflected LT differences in the same direction as the on-line results. Only data from the off-line coding were used in the analyses.

\section{Results}

The LTs for each infant were computed for (1) the preand posttests and (2) the same and change trials. A 2 (Age group: $10-12$ months vs. $6-8$ months) $\times 2$ (Test: pretest vs. posttest) repeated-measures ANOVA was conducted to determine if there were significant agerelated differences in the infants' interest in the task. There was no significant effect of Age group or Test $\left[\right.$ Mean $_{\text {PRETEST }}=12.77 \mathrm{~s}, \quad$ Mean $\left._{\text {POSTTEST }}=12.59 \mathrm{~s}\right]$, suggesting that infants maintained interest throughout the entirety of the experiment.

Infants' average LTs to same and change trials according to age group are plotted in Figure 2. The plot shows that both groups of infants looked longer to change trials than same trials.

Discrimination of the [ma]-[na] distinction was analyzed in a 2 (Age group: 10-12 months vs. 6-8 months) $\times 2$ (Habituating stimuli: [ma] vs. [na]) $\times 2$ (Test trials: same vs. change) repeated-measures ANOVA. There was a significant main effect of Test trials showing that infants looked significantly longer to change trials than they did to same trials $\left[\right.$ Mean $_{\text {SAME }}=3.93 \mathrm{~s}$, Mean $_{\text {CHANGE }}=6.02 \mathrm{~s} ; F(1,67)=$ 19.31, $p<.001$; partial $\eta^{2}=0.408$, observed power $=$ $0.989]$. Across both age groups 27 of the 32 infants tested looked longer to change trials than same trials. All 16 of the 6-8-month infants looked longer to change trials, while 11 of the 16 10-12-month-olds looked longer to change. There were no significant effects of Age group, Habituating stimuli, or their interaction.

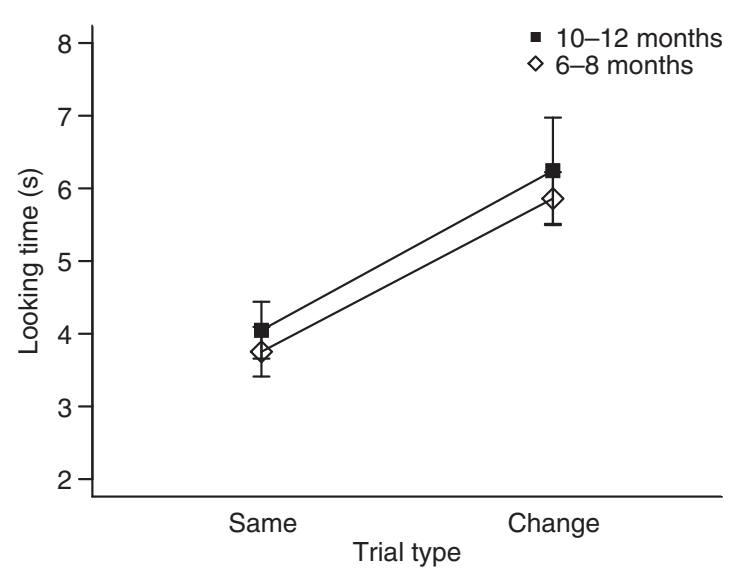

Figure 2 English-hearing infants' (at 10-12 and 6-8 months) looking time to same vs. change trials for the native [ma]-[na] contrast. Error bars represent standard error.

\section{Discussion}

That infants looked significantly longer to change trials than to same trials indicates that English-learning infants in the second half of their first year discriminate the native nasal place distinction [ma]-[na]. These results are consistent with the one other investigation into Englishhearing infants' perception of this contrast (Hillenbrand, 1984) and were expected: [ma] and [na] exhibit consistent differences in $\mathrm{F} 2 \times \mathrm{F} 3$ space and should be discriminable for young infants as well as for older infants who are exposed to this native contrast in the ambient language. Importantly, the present findings show that the $\mathrm{VH}$ procedure is sensitive enough to reveal discrimination of place of articulation within the nasal manner. As Hillenbrand's study employed a different task, a finding inconsistent with his could have potentially been due to methodological factors.

The results of Experiment 1 laid the foundation for Experiment 2, which addressed the question of how infants perceive a non-native nasal place contrast that is acoustically less salient than the [ma]-[na] distinction.

\section{Experiment 2}

The second experiment was designed to determine whether the perception of the non-native [na]-[na] distinction by English-learning infants at 6-8 and 10-12 months follows the perceptual pattern shown in numerous other studies of infant perception of consonants, whereby discrimination performance declines in later infancy in the absence of specific language experience. If it does, then 6-8-month-olds would be expected to discriminate the contrast, but 10-12-month-olds would not. Alternatively, if the similarity of [n] and [n] in F2 $\times$ F3 space affects the developmental trajectory, then both age groups are expected to have difficulty with the distinction. As in Experiment 1, two age groups of infants were tested 
using methods consistent with previous literature showing age and experience effects on non-native discrimination between 6 and 12 months (Werker \& Tees, 1984; Polka et al., 2001; Anderson et al., 2003; Kuhl et al., 2006; Burns et al., 2007).

\section{Methods}

The procedure and setup for Experiment 2 were identical to those in Experiment 1. The trials consisted of [na] and [na] tokens, whose acoustic characteristics are given in Table 1. The [na] stimuli were the same as those used in Experiment 1. The tokens were assembled in a fashion parallel to that described in the Stimuli section.

\section{Participants}

As in Experiment 1, infants were recruited from the database at the University of British Columbia. Data from 16 10-12-month-olds (eight females, eight males) and 16 6-8-month-olds (eight females, eight males) were analyzed. In order to achieve this set of 32, 51 infants were tested. Among the 10-12-month-old infants, 14 babies were not included in the analysis for the following reasons: crying or fussiness $(n=5)$, parental or caregiver interference $(n=1)$, experimental error $(n=1)$, habituating in six trials $(n=6)$, and failure to habituate $(n=1)$. Among the 6-8-month-old infants, four babies were not included due to parental or caregiver interference $(n=1)$, repeated test trials (LT to a test trial $<1$ second) $(n=1)$, habituating in six trials $(n=1)$, and failure to habituate $(n=1)$. As in Experiment 1 , all infants who participated were being exposed to at least 90\% Canadian English according to parental reports.

\section{Results}

Infants' attention to the $\mathrm{VH}$ task was assessed in a 2 (Age group: 10-12 months vs. 6-8 months) $\times 2$ (Test: Pretest vs. Posttest) repeated-measures ANOVA. There were no significant effects of Age group, Test, or their interaction, confirming that infants maintained interest in the task over the course of the experiment $\left[\right.$ Mean $_{\text {Pretest }}=12.74 \mathrm{~s}$, Mean $\left._{\text {Posttest }}=12.49 \mathrm{~s}\right]$.

Figure 3 plots the results of infants' LTs to same and change trials according to age group. Infants' discrimination of the non-native contrast was analyzed in a 2 (Age group: 10-12 and 6-8 months) $\times 2$ (Habituating stimuli: [na] vs. [ya]) $\times 2$ (Trial type: same vs. change) repeated-measures ANOVA. The analysis revealed no significant effects of Age group, Habituating stimuli, Trial type, or any interactions $\left[\right.$ Mean $_{\mathrm{SAmE}}=$ $5.04 \mathrm{~s}$, Mean $\left._{\text {ChAnge }}=4.66 \mathrm{~s}\right]$. Across both age groups, 10 of the 32 infants tested (six 6-8-month-olds and four 10-12-month-olds) looked longer to change trials than same trials. The lack of a significant difference in LT to same vs. change test trials indicates that infants across

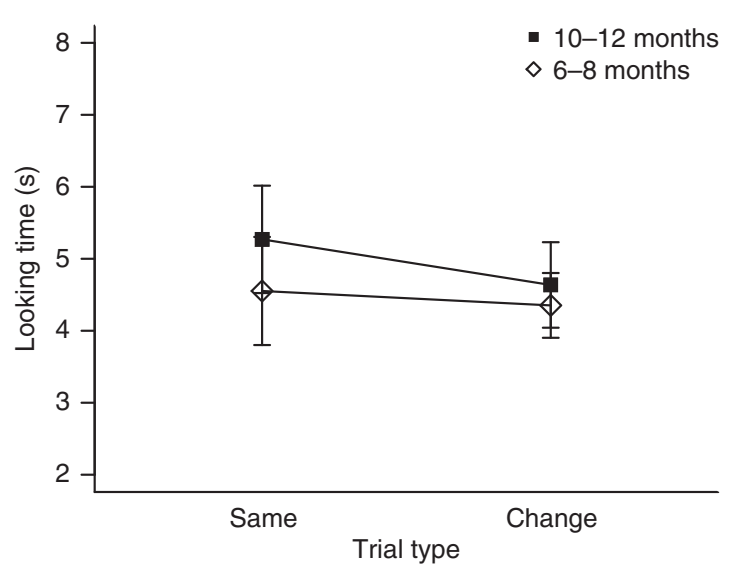

Figure 3 English-hearing infants' (at 10-12 and 6-8 months) looking time to same vs. change trials for the non-native [na][na] contrast. Error bars represent standard error.

the ages of 6-8 and 10-12 months did not reliably discriminate the non-native contrast.

\section{Discussion}

The results of Experiment 2 do not show the characteristic pattern of decline in discriminability of a non-native consonant contrast between 6-8 months and $10-12$ months. Although virtually all previous studies have shown that infants of 6-8 months are capable of discriminating non-native consonant contrasts, including those which adult listeners have difficulty discriminating (e.g. Trehub, 1976; Werker \& Tees, 1984; Polka et al., 2001), in this study English-learning 6-8-month-olds and, as expected, 10-12-month-olds - did not reliably show discrimination of [na]-[na]. That neither group of infants reliably discriminated [na]-[na] is consistent with an acoustic salience interpretation, which predicts that the acoustically more similar contrast would be perceived less accurately than the acoustically robust contrast (in this case, [ma]-[na]).

An alternative explanation for the results of Experiment 2 is that nasal place perception develops on a time course different from other consonants. That is, perhaps the more typical reorganization pattern, whereby infants develop from being language-general to language-specific perceivers, occurs earlier for nasals. This line of reasoning is further motivated by the finding that infants' perception of vowels indicates development of native-language sensitivity by $6-8$ months rather than the 10-12 months for oral consonants. Infants at 4 months are able to discriminate non-native vowel contrasts that they no longer discriminate at 68 months (Kuhl et al., 1992; Polka \& Werker, 1994). Nasals share acoustic properties with vowels, such as sustainable low-frequency resonance or sonority, which are different from those of most oral consonants. Such a similarity may hint at an earlier time course for an experientially induced decline. This possibility was explored in the next experiment. 


\section{Experiment 3}

That the younger infants in Experiment 2 failed to discriminate [na]-[na] suggests two possibilities. The first is that the developmental trajectory from early discrimination of non-native contrasts to decline in discriminability occurs earlier for nasal than for oral consonant contrasts. The second interpretation is that the results reflect the acoustic similarity between [na] and [na], shown to be operative in Filipino adults' perception of native [na]-[na] (Narayan, 2008). To disentangle these two interpretations, in Experiment 3 we tested 4-5month-old English-hearing infants on native [ma]-[na] and non-native [na]-[na]. If earlier perceptual decline of non-native contrasts applies to nasal place discrimination, infants in this very young age group are expected to successfully discriminate both contrasts.

\section{Methods}

The procedure and setup were identical to those in Experiments 1 and 2. The stimuli used in the present experiment are the same [ma], [na] and [na] tokens used in those experiments.

\section{Participants}

Data from 32 infants were analyzed in the study, 16 infants (eight females, eight males) tested in the [ma]-[na] group and 16 (seven females, nine males) in the [na]-[na] group. All of the infants were between the ages of 4 months, 0 days and 5 months, 10 days at the time of the study (mean $=147$ days, range $=135$ to 160 days). An additional 24 infants participated in the study, but had to be excluded from analysis due to crying or fussiness $(n=13)$, parental or caregiver interference $(n=2)$, experimental error $(n=1)$, habituation in six trials $(n=1)$, repeated test trials $(n=3)$, and failure to habituate $(n=4)$. As in Experiments 1 and 2, only infants who received at least $90 \%$ Canadian English input according to parental reports were recruited for participation.

\section{Results}

As in the earlier experiments, the results of a 2 (Contrast: [ma]-[na] vs. [na]-[na]) $\times 2$ (Test: pretest vs. posttest) repeated-measures ANOVA testing infants' attention in the VH task $\left(\right.$ Mean $_{\text {PRETEST }}=12.54 \mathrm{~s}$, Mean $_{\text {PostTEST }}=$ $12.36 \mathrm{~s})$ showed no effect of either Contrast or Test, suggesting that infants maintained attention throughout the task.

Figure 4 plots infants' LTs to same vs. change in the two contrast conditions. Because [na] tokens were used as habituating stimuli for both groups ([ma]-[na] and [na]-[na]), infants were coded with a four-level betweensubjects factor (Habituation-Contrast: within the [ma][na] group, habituation with [ma]; within the [ma]-[na]

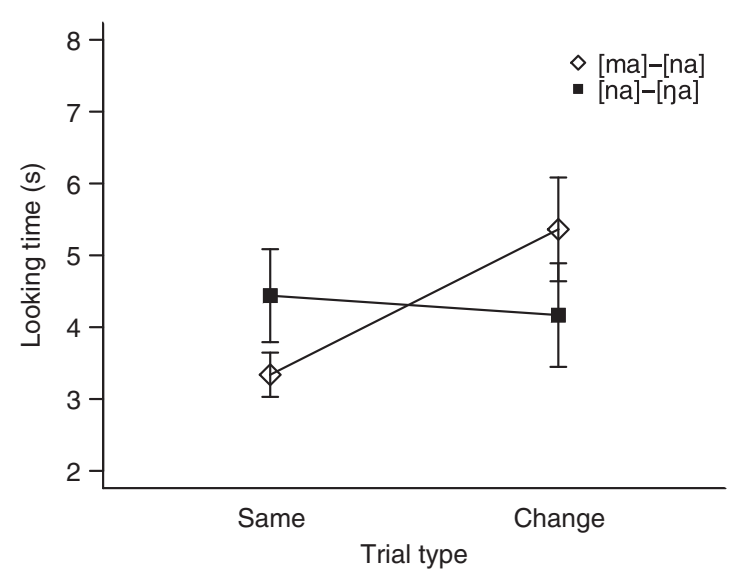

Figure 4 English-hearing infants' (at 4-5 months) looking time to same vs. change trials for the native [ma]-[na] and nonnative [na]-[ya] contrasts. Error bars represent standard error.

group, habituation with [na]; within the [na]-[ya] group, habituation with [na]; and within the [na]-[na] group, habituation with [na]). Discrimination of the two contrasts was examined in a 4 (Habituation-Contrast) $\times$ 2 (Trial type: same vs. change) ANOVA. The analysis showed a main effect of Trial type, with infants looking significantly longer to change trials than same trials $\left[F(1,28)=6.41, p<.05 ;\right.$ partial $\eta^{2}=0.186$, observed power $=0.686]$. There was also a significant interaction between Trial type and Habituation-Contrast $[F(1$, 28) $=3.98, \quad p<.05 ;$ partial $\eta^{2}=0.299, \quad$ observed power $=0.779$ ]. This interaction was further probed with two post-hoc 2 (Trial type) $\times 2$ (Habituating stimuli) repeated-measures ANOVAs. In the [ma]-[na] condition there was a significant effect of Trial type with infants looking longer to change trials than same trials $\left[\operatorname{Mean}_{\mathrm{SAME}}=3.34 \mathrm{~s}, \operatorname{Mean}_{\mathrm{CHANGE}}=5.36 \mathrm{~s} ; F(1,14)=\right.$ $12.20, p<.005$; partial $\eta^{2}=0.466$, observed power $=$ $0.901]$, and this pattern held for 13 of the 16 infants. There was no significant interaction between Trial type and Habituating stimuli. There was also no effect of Habituating stimuli; consequently, the interaction between Trial type and Habituation-Contrast was driven by the effect of contrast on LT to same and change. This is evident in Figure 4.

In the [na]-[na] condition, there was no effect of Trial type nor an interaction between Trial type and Habituating stimuli $\left[\right.$ Mean $_{\mathrm{SAME}}=4.44 \mathrm{~s}$, Mean $_{\text {ChANGE }}=$ $4.17 \mathrm{~s}$ ]. Six of the 16 infants in the [na]-[ya] group looked longer to change trials than same trials. The interaction in the main analysis was driven by infants' discrimination of the native contrast.

\section{Discussion}

The pattern of discrimination exhibited by 4-5-monthold infants is similar to the patterns shown by 6-8- and 10-12-month-old infants in Experiments 1 and 2. That the 4-5-month-olds, like the older infants, did not discriminate the non-native, acoustically more similar 
[na]-[ya] difference argues against an interpretation of early decline due to lack of ambient language experience as being responsible for the pattern of nasal place perception observed in Experiments 1 and 2. Moreover, that English-learning infants, across their first year, successfully discriminated native [ma]-[na] suggests that the lack of discrimination of [na]-[na] was not due to a general inability to discriminate nasal place of articulation. Rather the discrimination pattern shown by the English-learning infants across the three experiments points toward the acoustic properties of the non-native contrast itself - in particular, we claim, toward the acoustic similarity of these nasals.

However, strong evidence in support of the acoustic salience interpretation requires a cross-language design in which discrimination of [na]-[na] is shown to be difficult for young Filipino-learning infants. In Experiment 4 we present the control case to the nonnative English-learning studies by testing Filipinolearning infants on the native [na]-[na] distinction.

\section{Experiment 4}

The results of Experiments 2 and 3 raise the question of whether any group of infants would reliably discriminate [na]-[na]. Previous research overwhelmingly suggests that infants home in on the linguistically relevant consonant contrasts from their ambient language by the end of their first year. English-learning infants' inability to discriminate (using the VH paradigm) [na]-[na] opens up the possibility, if not likelihood, that Filipino 6-8month-olds might also not discriminate these acoustically similar sounds of Filipino. Moreover, it may be that less robust phonetic contrasts are on a relatively slow developmental time course; if so, perhaps even 10-12-month-old Filipino-learning infants will not discriminate [na]-[na]. There is, as discussed in the Introduction, already evidence in the literature that not all contrasts are on the same time course, with vowel perception showing language differentiation by as early as 6 months (e.g. Kuhl et al., 1992) and the consonantal $/ \mathrm{d} /-/ \mathrm{d} /$ contrast not showing language-specific patterning until after 12 months (Polka et al., 2001; Sundara et al., 2006). Given these patterns of results, a possible developmental profile in the present experiment is one in which infants' perception of a native acoustically less salient contrast is discriminated only in later infancy.

\section{Methods}

\section{Participants}

Data from 28 Filipino-hearing infants were analyzed in the study (12 10-12-month-olds and 16 6-8-month-olds). The older group (two females, 10 males) were between the ages of 10 months, 1 day and 12 months, 20 days (mean $=339$ days, range $=302$ to 380 days $)$. The younger group (eight females, eight males) were between the ages of 6 months, 4 days and 8 months, 22 days (mean $=219$ days, range $=188$ days to 262 days). An additional 17 infants participated in the study, but had to be excluded from analysis due to their failure to habituate $(n=2)$, habituating in six trials $(n=2)$, crying/fussiness $(n=10)$, refusing to look at the TV monitor $(n=1)$, repeated test trials $(n=1)$, and experimental error $(n=1)$.

Many Filipinos in the Vancouver area speak Filipino as well as other Austronesian languages of the Philippines. For many families, Filipino is used as a lingua franca within larger Filipino social networks, while an ethnically and regionally specific Austronesian language, such as Cebuano or Ilocano, is used at home. Infants who were exposed to any Austronesian language of the Philippines were allowed to participate in the study, as long as they met the exposure criterion described below. ${ }^{4}$ All of the Austronesian languages of the Philippines show a robust, contrastive presence of the velar, alveolar and bilabial nasal places in syllable-onset position.

In order to assess the amount of infants' Filipino exposure, a brief language-background questionnaire was administered to the accompanying parent/ guardian. This questionnaire was similar to those used by infant speech perception labs interested in quantifying the level of bilingualism in a particular household (e.g. Bosch \& Sebastián-Gallés, 2003). Only infants who received at least $50 \%$ exposure to a Filipino language and whose mother was born and raised in the Philippines and was a native speaker of a Filipino language were recruited for participation.

\section{Setup, procedure and stimuli}

The setup and procedure were identical to those in Experiments 1,2 and 3. The stimuli used in the present experiment were the same [na] and [na] tokens used in Experiments 2 and 3.

\section{Results}

A 2 (Age group: $6-8$ vs. $10-12$ months) $\times 2$ (Test: pretest vs. posttest) repeated-measures ANOVA testing infants' attention in the VH task $\left(\right.$ Mean $_{\text {Pretest }}=12.51 \mathrm{~s}$, Mean $_{\text {Postтest }}=12.53 \mathrm{~s}$ ) showed no effect of either Age or Test, suggesting that infants maintained attention throughout the task. Figure 5 plots infants' average looking time to same and change trials according to age group. The plot clearly shows that infants in the older group look reliably longer to change than same trials,

\footnotetext{
${ }^{4}$ Restricting the subject pool to infants being exposed to only Filipino (Tagalog) would have resulted in an extraordinarily long experiment completion time as Tagalogs make up a small percentage of Vancouverarea Filipinos.
} 


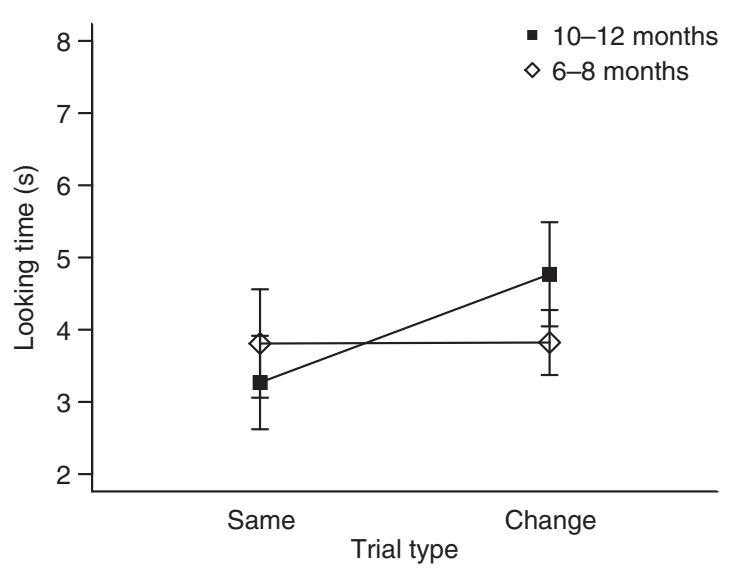

Figure 5 Filipino-learning infants' (at 6-8 and 10-12 months) looking time to same vs. change trials for the native [na]-[na]. Error bars represent standard error.

while infants in the younger group look equally long to same and change trials.

In order to assess whether infants attended to the visual stimulus across the duration of the task, a 2 (pretest vs. posttest LT) $\times 2$ (Age group: $10-12$ vs. 6 8 months) mixed ANOVA was conducted. As in the other experiments, there was no effect of either test type or age group, suggesting that infants maintained attention throughout the task.

Discrimination results were analyzed in a 2 (Age group: $10-12$ vs. $6-8$ months) $\times 2$ (Habituating stimuli: [na] vs. [na] $\times 2$ (Trial type: same vs. change) ANOVA. The analysis showed a main effect of Trial type $\left[F(1,24)=8.64, p<.01 ;\right.$ partial $\eta^{2}=0.265$, observed power $=0.805]$ with infants looking significantly longer overall to change trials than same trials. The effect of Trial type can only be interpreted in light of its significant interaction with Age group $[F(1,24)=8.34$, $p<.01 ;$ partial $\eta^{2}=0.258$, observed power $\left.=0.791\right]$. The interaction was further probed with a series of paired samples $t$-tests. Within the 6-8-month group, there was no significant difference in infants' looking time to same and change trials $\left[\mathrm{Mean}_{\mathrm{SAME}}=3.81 \mathrm{~s}\right.$, $\left.\operatorname{Mean}_{\text {CHANGE }}=3.82 \mathrm{~s} ; t(15)=-0.042, n s\right]$. Seven out of the 16 infants in the 6-8-month-old group looked longer to change trials than same trials. There was a significant difference in looking time to same vs. change trials for 10-12-month-olds, with infants looking longer to change trials than same trials $\left[\mathrm{Mean}_{\mathrm{SAME}}=3.26 \mathrm{~s}, \mathrm{Mean}_{\mathrm{CHANGE}}=\right.$ $4.77 \mathrm{~s} ; t(11)=-3.57, p<.005$, Cohen's $d=0.884]$. Eleven of the 12 infants in the 10-12-month-old group looked longer to change trials than same trials.

\section{Discussion}

Filipino-hearing infants showed discrimination of [na][na] only towards the end of the first year of life. This perceptual development of a native contrast, from poor discrimination in young infancy to successful discrimination in later infancy, is different from the typical pattern in which infants discriminate phonetic contrasts early in their first year and the effect of native language exposure later in the first year is to maintain or improve discriminability to adult-like levels. The current findings suggest that some native consonant contrasts are difficult for young infants to resolve, with nativelanguage experience being necessary to separate the acoustically difficult contrast into perceptually relevant categories.

The results of the present experiment, together with those of Experiments 1-3, exhibit the combined effects of acoustic salience and language experience on discrimination of nasal place contrasts in infancy. Aspects of these findings were tentatively predicted on the basis of phonological typology data, acoustic measures, and findings from experiments with adult listeners described in the Introduction.

\section{General discussion}

The experiments in this study were designed to explore the developmental perception of nasal place contrasts whose members differ in acoustic separation along the perceptually relevant $\mathrm{F} 2 \times \mathrm{F} 3$ dimension. Robustness of acoustic differences is known to influence adults' discrimination of non-native phonetic contrasts (e.g. Larkey et al., 1978; Polka, 1991; Pruitt, 1995; Harnsberger, 2001). Moreover, as discussed in the Introduction, contrasts based on less salient differences tend, very broadly speaking, to be among the less common contrasts in phonological systems (e.g. Maddieson, 1984; Lindblom, 1986). The typological asymmetry of nasal place contrasts, with alveolar and bilabial nasals being found more often than velar nasals in the world's languages, especially in syllable-onset position, combined with results showing that adult discrimination of [ma]-[na] is significantly better than discrimination of [na]-[ya] even in cases where both contrasts are native (Narayan, 2008), led us to hypothesize in the present study that discrimination of [na]-[ya] would be difficult for young infants regardless of their language experience.

The findings strongly suggest that the perceptual development of nasal place discrimination is influenced by the extent - which we have defined in acoustic terms of the differences between the place categories. Englishlearning infants at all of the tested ages (4-12 months) successfully discriminated the acoustically salient [ma] [na] difference, but not the less salient [na]-[na]. Further, Filipino-learning infants showed discrimination of [na] [na] at 10-12 months but not the at 6-8 months. The Filipino-learning infants' results suggested that, even when the less salient difference $([\mathrm{n}]-[\mathrm{n}])$ is present in the ambient language, experience beyond the first half year of life is required for the infant to successfully discriminate the target sounds (as measured by the visual habituation paradigm). The effects of specific language experience are evident by the end of the first 
year, however, indicating that the time course of language-specific development for this nasal contrast is similar to the time course for the decline in sensitivity to non-native contrasts and facilitation of native contrasts observed for other consonants. That is, infants' discrimination of most consonant contrasts shows differentiation according to native language experience by 12 months of age (but see Polka et al., 2001), with differentiation of less salient contrasts being facilitated with increased native language exposure.

Thus far, we have characterized the robustness of a phonetic contrast in acoustic terms. Two prominent theories of speech perception, Motor Theory (Liberman \& Mattingly, 1985) and Direct Realism (Fowler, 1986, 1996; Best, 1995) recognize gestures as the perceptual primitives, raising the question of whether the current findings are equally suggestive of the role of articulatory robustness in perceptual development. There is not a simple answer to this question because articulatory similarity, like acoustic similarity, can be defined along a number of parameters. However, Articulatory Phonology, which analyzes vocal tract behaviors in terms of the constricting actions or gestures of six distinct vocal organs - the lips, tongue tip, tongue body, tongue root, velum and larynx (e.g. Browman \& Goldstein, 1992) - offers especially clear perceptual predictions based on gesturally defined similarity. Goldstein and Fowler (2003; also Goldstein, Byrd \& Saltzman, 2006), for example, predict that phonetic contrasts between sounds produced with distinct organs will be accurately perceived by infants, while contrasts between sounds produced with the same organ will be less accurately perceived. Best and McRoberts' (2003) tests of this theory with several phonetic contrasts provide supporting evidence. The present data, however, do not readily lend themselves to a gesturally based interpretation in that members of both distinctions, [ma]-[na] (lips vs. tongue tip/blade) and [na]-[na] (tongue tip/blade vs. tongue body), are produced with organs that form constrictions independently of each other.

In situating these results for nasal place discrimination within the broad context of the development of speech perception, an important issue to be addressed is the relative contribution of native-language experience and acoustic salience in infancy and beyond. Aslin and Pisoni's (1980) attunement theory (see Introduction) posits that discriminative capabilities that are partially present or broadly specified initially are maintained or sharpened by relevant linguistic experience, which facilitates discrimination (e.g. Polka et al., 2001; Kuhl et al., 2006, Sundara et al., 2006; Tsao et al., 2006) and realigns initial sensitivities (Burns et al., 2007). In the absence of relevant experience, some contrasts remain discriminable (e.g. Best et al., 1988; Polka \& Bohn, 1996) while discrimination of others becomes attenuated (e.g. Werker \& Tees, 1984; Anderson et al., 2003). We interpret the present results as likewise consistent with attunement theory. Specifically, we claim that young infants in the present study show evidence for nasal place discrimination, but that their responses reflect broadly tuned acoustic sensitivity. That is, we take the scope of the analysis to be nasal place contrasts, with a relevant acoustic property being distance in F2 $\times$ F3 space (as in the plot of Filipino [m], [n], and [n] tokens in Figure 1). That young infants show discrimination of [ma]-[na] but not [na]-[ya] suggests that infants' initial specification of nasal place perception broadly reflects sounds like [m] and [n], which are well separated especially along the F2 dimension, while [na] and [na] are initially indistinct. ([m] and [y] are also clearly separated in F2 $\times$ F3 space, and we would expect young infants to discriminate this contrast as well, although this was not tested.) With specific language experience, the discrimination of finergrained phonetic contrasts is facilitated.

We fully expect that the developmental profile described by the present set of studies is not unique to nasal place contrasts, and predict that a similar profile should hold for other contrasts with comparable salience differences. Keeping in mind that it is difficult to compare salience across different acoustic properties (unlike the present study which assessed relative salience of nasal contrasts within the same acoustic space), it does appear that some highly salient contrasts show developmental patterns different from less salient contrasts. As discussed in the Introduction, distinctive non-native click contrasts remain discriminable across development and into adulthood for English speakers (Best et al., 1988). In addition, non-native vowel contrasts may remain discriminable across development for acoustic reasons (Polka \& Bohn, 1996). There are periods in development in which vowels in an acoustically crowded space are poorly discriminated by infants for whom the contrast is native (Bosch \& Sebastián-Gallés, 2003; Sebastián-Gallés \& Bosch, in press). Moreover, Polka et al. (2001) suggest that the lack of an effect of language experience at 10-12 months for Canadian English- and Canadian French-learning infants' perception of the English $/ \mathrm{d} /-/ ठ /$ contrastmay be due to the weak acoustic properties of [ð]. While such results do not show the developmental profile shown in the current set of studies with nasals, they tentatively point in the direction of a more general effect of differences in acoustic salience in infant speech perception.

Infants in the present study have shown that the generality of early discriminative abilities can be tempered by the perceptual realities exhibited by acoustically similar contrasts. In addition, our results highlight the importance of the 10-12-month period in establishing the phonetic framework for the infant's emerging phonology and higher level language learning (Werker \& Yeung, 2005). But how does the infant resolve difficult acoustics like the [n]-[n] distinction in the present study? Our current work is exploring infants' sensitivities to stochastic distributions in the speech signal (Maye, Werker \& Gerken, 2002; Maye, Weiss \& Aslin 2008) as well as their budding knowledge of a link between speech and concept (e.g. Waxman \& Booth, 
2003) as potential mechanisms promoting the re-focusing of attention to finer-grained aspects of the acoustic signal which were otherwise overshadowed in early infancy.

\section{Acknowledgements}

This research was supported by a fellowship from the Rackham Graduate School at the University of Michigan to CRN and a grant from the National Sciences and Engineering Research Council of Canada to JFW. This work appears as part of the first author's dissertation submitted to the Department of Linguistics at the University of Michigan. Thanks to members of the Infant Studies Centre (UBC), the Phonetics/Phonology discussion group (University of Michigan), and audiences at the Boston University Conference on Language Development (BUCLD30), LabPhon10, and the Linguistic Society of America annual meeting (2007) for their helpful comments. Special thanks to Corinna Elliott and Vivian Pan for testing some of the infants, and to Ramesh Thiruvengadaswamy and Laurel Fais for technical assistance. Thanks also to Vashti Garcia and Marisa Cruickshank for recruiting the infants in this study. This work benefited from the thoughtful and careful comments of three anonymous reviewers.

\section{References}

Anderson, G.D.S. (2005). The velar nasal (N). In M. Haspelmath, M.S. Dryer, D. Gil, \& B. Comrie (Eds.), The world atlas of language structures (pp. 42-45). Oxford: Oxford University Press.

Anderson, J.L., Morgan, J.L., \& White, K.S. (2003). A statistical basis for speech sound discrimination. Language and Speech, 46 (2-3), 155-182.

Aslin, R.N., \& Pisoni, D.B. (1980). Some developmental processes in speech perception. In G.H. Yeni-Komshian, J.F. Kavanagh, \& C.A. Ferguson (Eds.), Child phonology (Vol. 2: Perception, pp. 67-94). New York: Academic Press.

Aslin, R.N., Pisoni, D.B., Hennessy, B.L., \& Perey, A.J. (1981). Discrimination of voice-onset time by human infants: new findings and implications for the effects of early experience. Child Development, 52, 1135-1145.

Baker, S.A., Michnick-Golinkoff, R., \& Petitto, L.A. (2006). New insights into old puzzles from infants' categorical discrimination of soundless phonetic units. Language Learning and Development, 2 (3), 147-162.

Best, C. (1995). A direct realist view of cross-language speech perception. In W. Strange (Ed.), Speech perception and linguistic experience: Issues in cross-language research (pp. 171204). Baltimore, MD: York Press.

Best, C., \& McRoberts, G.W. (2003). Infant perception of nonnative consonant contrasts that adults assimilate in different ways. Language and Speech, 46 (2-3), 183-216.

Best, C.T., McRoberts, G.W., LaFleur, R., \& Silver-Isenstadt, J. (1995). Divergent developmental patterns for infants' perception of two nonnative consonant contrasts. Infant Behavior and Development, 18 (3), 339-350.
Best, C.T., McRoberts, G.W., \& Sithole, N.M. (1988). Examination of perceptual reorganization for nonnative speech contrasts: Zulu click discrimination by English-speaking adults and infants. Journal of Experimental Psychology: Human Perception and Performance, 14 (3), 345-360.

Bosch, L., \& Sebastián-Gallés, N. (2003). Simultaneous bilingualism and the perception of a language-specific vowel contrast in the first year of life. Language and Speech, $\mathbf{4 6}$ (2-3), 217-243.

Browman, C.P., \& Goldstein, L. (1992). Articulatory phonology: an overview. Phonetica, 49, 155-180.

Burnham, D. (1986). Developmental loss of speech perception: exposure to and experience with a first language. Applied Psycholinguistics, 7, 207-239.

Burns, T.C., Yoshida, K.A., Hill, K., \& Werker, J.F. (2007). Bilingual and monlingual infant phonetic development. Applied Psycholinguistics, 28 (3), 455-474.

Cheour, M., Alho, K., Ceponiene, R., Reinikainen, K., Sainio, K., Pohjavuori, M., Aaltonen, O., \& Naatanen, R. (1998). Maturation of mismatch negativity in infants. International Journal of Psychophysiology, 29, 217-226.

Cohen, L.B., Atkinson, D.J., \& Chaput, H.H. (2004). Habit X: $A$ new program for obtaining and organizing data in infant perception and cognition studies (Version 1.0). Austin, TX: University of Texas.

Eilers, R.E., Wilson, W.R., \& Moore, J.M. (1977). Developmental changes in speech discrimination in infants. Journal of Speech and Hearing Research, 20, 766-780.

Eimas, P.D., Siqueland, E.R., Jusczyk, P.W., \& Vigorito, J. (1971). Speech perception in infants. Science, 171, 303-306.

Fowler, C.A. (1986). An event approach to the study of speech perception for a direct-realist perspective. Journal of Phonetics, 14, 3-28.

Fowler, C.A. (1996). Listeners do hear sounds, not tongues. Journal of the Acoustical Society of America, 99, 1730-1741.

Goldstein, L., Byrd, D., \& Saltzman, E. (2006). The role of vocal tract gestural action units in understanding the evolution of phonology. In M. Arbib (Ed.), Action to language via the mirror neuron system (pp. 215-249). Cambridge: Cambridge University Press.

Goldstein, L., \& Fowler, C.A. (2003). Articulatory phonology: a phonology for public language use. In N. Schiller \& A. Meyer (Eds.), Phonetics and phonology in language comprehension and production (pp. 159-297). Berlin: Mouton de Gruyer.

Harnsberger, J.D. (2001). The perception of Malayalam nasal consonants by Marathi, Punjabi, Tamil, Oriya, Bengali, and American English listeners: a multidimensional scaling analysis. Journal of Phonetics, 29, 303-327.

Hillenbrand, J. (1984). Speech perception by infants: categorization based on nasal consonant place of articulation. Journal of the Acoustical Society of America, 75 (5), 1613-1622.

Jusczyk, P.W. (1997). The discovery of spoken language. Cambridge, MA: MIT Press.

Kajikawa, S., Fais, L., Mugitani, R., Werker, J.F., \& Amano, S. (2006). Cross-language sensitivity to phonotactic patterns in infants. Journal of the Acoustical Society of America, 120 (4), 2278-2284.

Kuhl, P.K. (2004). Early language acquisition: cracking the speech code. Nature Reviews Neuroscience, 5 (11), 831-841.

Kuhl, P.K., Stevens, E., Hayashi, A., Deguchi, T., Kiritani, S., \& Iverson, P. (2006). Infants show a facilitation effect for native language phonetic perception between 6 and 12 months. Developmental Science, 9 (2), 13-21. 
Kuhl, P.K., Williams, K.A., Lacerda, F., Stevens, K.N., \& Lindblom, B. (1992). Linguistic experience alters phonetic perception in infants by 6 months of age. Science, 255, 606-608.

Larkey, L.S., Wald, J., \& Strange, W. (1978). Perception of synthetic nasal consonants in initial and final syllable position. Perception and Psychophysics, 23 (4), 299-312.

Liberman, A.M., \& Mattingly, I.G. (1985). The motor theory of speech perception revised. Cognition, 21, 1-36.

Liljencrants, J., \& Lindblom, B. (1972). Numerical simulation of vowel quality: the role of perceptual contrast. Language, 48 (4), 839-862.

Lindblom, B. (1986). Phonetic universals in vowel systems. In J.J. Ohala \& J.J. Jaeger (Eds.), Experimental phonology (pp. 13-44). Orlando, FL: Academic Press.

McMurray, B., \& Aslin, R.N. (2005). Infants are sensitive to within-category variation in speech perception. Cognition, $\mathbf{9 5}$, B15-B26.

Maddieson, I. (1984). Patterns of sounds. Cambridge: Cambridge University Press.

Mattock, K., \& Burnham, D. (2006). Chinese and English infants' tone perception: evidence for perceptual reorganization. Infancy, 10 (3), 241-265.

Maye, J., Werker, J.F., \& Gerken, L. (2002). Infant sensitivity to distributional information can affect phonetic discrimination. Cognition, 82, B101-B111.

Maye, J., Weiss, D.J., \& Aslin, R.N. (2008). Statistical phonetic learning in infants: facilitation and feature generalization. Developmental Science, 11 (1), 122-134.

Narayan, C.R. (2008). The acoustic-perceptual salience of nasal place contrasts. Journal of Phonetics, 36, 191217.

Nittrouer, S., \& Miller, M.E. (1997). Predicting developmental shifts in perceptual weighting schemes. Journal of the Acoustical Society of America, 101 (4), 2253-2266.

Pegg, J.E., Werker, J.F., \& McLeod, P.J. (1992). Preference for infant-directed over adult-directed speech: evidence from 7week-old infants. Infant Behavior and Development, 15, 325345.

Pisoni, D. (1977). Identification and discrimination of the relative onset time of two component tones: implications for voicing perception in stops. Journal of the Acoustical Society of America, 61 (5), 1352-1362.

Polka, L. (1991). Cross-language speech perception in adults: phonemic, phonetic, and acoustic contributions. Journal of the Acoustical Society of America, 89, 2961-2977.

Polka, L., \& Bohn, O.-S. (1996). A cross-language comparison of vowel perception in English-learning and German-learning infants. Journal of the Acoustical Society of America, 100 (1), 577-592.

Polka, L., Colantonio, C., \& Sundara, M. (2001). A crosslanguage comparison of $/ \mathrm{d} /-/ \mathrm{\partial} /$ perception: evidence for a new developmental pattern. Journal of the Acoustical Society of America, 109 (5), 2190-2201.

Polka, L., \& Werker, J.F. (1994). Developmental changes in perception of nonnative vowel contrasts. Journal of Experimental Psychology: Human Perception and Performance, 20 (2), 421-435.

Pruitt, J.S. (1995). The perception of Hindi dental and retroflex stop consonants by native speakers of Japanese and American English. Unpublished doctoral dissertation, University of South Florida, Tampa.
Rivera-Gaxiola, M., Silva-Pereyra, J., \& Kuhl, P.K. (2005). Brain potentials to native and non-native speech contrasts in 7- and 11-month-old American infants. Developmental Science, 8 (2), 162-172.

Saffran, J.R., Werker, J.F., \& Werner, L.A. (2006). The infant's auditory world: hearing, speech, and the beginnings of language. In R. Siegler \& D. Kuhn (Eds.), Handbook of child development (6th edn., pp. 58-108). New York: Wiley.

Sebastián-Gallés, N. (2005). Cross-language speech perception. In D.B. Pisoni \& R. Remez (Eds.), The handbook of speech perception (pp. 546-566). Oxford: Blackwell.

Sebastián-Gallés, N. (2006). Native-language sensitivities: evolution in the first year of life. Trends in Cognitive Sciences, 10 (6), 239-241.

Sebastián-Gallés, N., \& Bosch, L. (in press). Developmental shift in the discrimination of vowel contrasts in bilingual infants: is the distributional account all there is to it? Developmental Science.

Strange, W. (1995). Cross-language studies of speech perception: a historical review. In W. Strange (Ed.), Speech perception and linguistic experience: Issues in cross-language research (pp. 3-45). Baltimore, MD: York Press.

Streeter, L.A. (1976). Language perception of two-month-old infants shows effects of both innate mechanisms and experience. Nature, 259, 39-41.

Sundara, M., Polka, L., \& Genesee, F. (2006). Languageexperience facilitates discrimination of $/ \mathrm{d}-ð /$ in monolingual and bilingual acquisition of English. Cognition, 100, 369-388.

Trehub, S.E. (1976). The discrimination of foreign speech contrasts by infants and adults. Child Development, 47, 466-472.

Tsao, F.M., Liu, H.M., \& Kuhl, P.K. (2006). Perception of native and non-native affricate-fricative contrasts: crosslanguage tests on adults and infants. Journal of the Acoustical Society of America, 120 (4), 2285-2294.

Tsushima, T., Takizawa, O., Sasaki, M., Shiraki, S., Nishi, K., Kohno, M., Menyuk, P., \& Best, C. (1994). Discrimination of English / $\mathrm{r}-\mathrm{l} /$ and /w-y/ by Japanese infants at 6-12 months: language-specific developmental changes in speech perception abilities. Paper presented at the International Conference on Spoken Language Processing.

Waxman, S.R., \& Booth, A.E. (2003). The origins and evolution of links between word learning and conceptual organization: new evidence from 11-month-olds. Developmental Science, 6 (2), 130-137.

Werker, J.F., Gilbert, J.H.V., Humphrey, K., \& Tees, R.C. (1981). Developmental aspects of cross-language speech perception. Child Development, 52, 349-353.

Werker, J.F., Shi, R., Desjardins, R., Pegg, J.E., Polka, L., \& Patterson, M. (1998). Three methods for testing infant speech perception. In A.M. Slater (Ed.), Perceptual development: Visual, auditory, and speech perception in infancy (pp. 389 420). London: UCL Press.

Werker, J.F., \& Tees, R.C. (1984). Cross-language speech perception: evidence for perceptual reorganization during the first year of life. Infant Behavior and Development, 7, 4963.

Werker, J.F., \& Yeung, H.H. (2005). Infant speech perception bootstraps word learning. Trends in Cognitive Sciences, 9 (11), 519-527.

Received: 17 July 2008

Accepted: 18 February 2009 